\title{
Modelling Protagonist Goals and Desires in First-Person Narrative
}

\author{
Elahe Rahimtoroghi, Jiaqi Wu, Ruimin Wang, \\ Pranav Anand and Marilyn A Walker \\ University of California Santa Cruz \\ Santa Cruz, CA, US \\ \{erahimto, jwu64, ruiwang, panand, mawalker\}@ucsc.edu
}

\begin{abstract}
Many genres of natural language text are narratively structured, a testament to our predilection for organizing our experiences as narratives. There is broad consensus that understanding a narrative requires identifying and tracking the goals and desires of the characters and their narrative outcomes. However, to date, there has been limited work on computational models for this problem. We introduce a new dataset, DesireDB, which includes goldstandard labels for identifying statements of desire, textual evidence for desire fulfillment, and annotations for whether the stated desire is fulfilled given the evidence in the narrative context. We report experiments on tracking desire fulfillment using different methods, and show that LSTM Skip-Thought model achieves F-measure of 0.7 on our corpus.
\end{abstract}

\section{Introduction}

Humans appear to organize and remember everyday experiences by imposing a narrative structure on them (Nelson, 1989; Thorne and Nam, 2009; Bruner, 1991; McAdams et al., 2006), and many genres of natural language text are therefore narratively structured, e.g. dinner table conversations, news articles, user reviews and blog posts (Polanyi, 1989; Jurafsky et al., 2014; Bell, 2005; Gordon et al., 2011). Moreover, there is broad consensus that understanding a narrative involves activating a representation, early in the narrative, of the protagonist and her goals and desires, and then maintaining that representation as the narrative evolves, as a vehicle for explaining the protagonist's actions and tracking narrative outcomes (Elson, 2012; Rapp and Gerrig, 2006; Trabasso

\begin{tabular}{|c|}
\hline $\begin{array}{l}\text { People did seem pleased to see me but all I [wanted to] } \\
\text { do was talk to a particular friend. }\end{array}$ \\
\hline $\begin{array}{l}\text { I'm off this weekend and had really [hoped to] get out } \\
\text { and dance. }\end{array}$ \\
\hline $\begin{array}{l}\text { We [decided to] just go for a walk and look at all the } \\
\text { sunflowers in the neighborhood. }\end{array}$ \\
\hline $\begin{array}{l}\text { I [couldn't wait to] get out of our cheap and somewhat } \\
\text { charming hotel and show James a little bit of Paris. }\end{array}$ \\
\hline $\begin{array}{l}\text { We drove for just over an hour and [aimed to] get to Trin- } \\
\text { ity beach to set up for the night. }\end{array}$ \\
\hline $\begin{array}{l}\text { She called the pastor, and he had time, too, so, we [ar- } \\
\text { ranged to] meet Saturday at 9am. }\end{array}$ \\
\hline $\begin{array}{l}\text { Even though my deadline wasn't until } 4 \text { p.m., I [needed } \\
\text { to] write the story as quickly as possible. }\end{array}$ \\
\hline
\end{tabular}

Figure 1: Desire expressions in personal narratives

and van den Broek, 1985; Lehnert, 1981).

To date, there has been limited work on computational models for recognizing the expression of the protagonist's goals and desires in narrative texts, and tracking their corresponding narrative outcomes. We introduce a new corpus DesireDB of $\sim 3,500$ first-person informal narratives with annotations for desires and their fulfillment status, available online. ${ }^{1}$ Because first-person narratives often revolve around the narrator's private states and goals (Labov, 1972), this corpus is highly suitable as a testbed for identifying human desires and their outcomes. Moreover, first-person narratives allow the narrative protagonist (first-person) to be easily identified and tracked. Figure 1 illustrates examples of desire and goal expressions in our corpus.

DesireDB is open domain. It contains a broad range of expressions of desires and goal statements in personal narratives. It also includes the narrative context for each desire statement as shown in Figure 2. We include both prior and

\footnotetext{
${ }^{1}$ https://nlds.soe.ucsc.edu/DesireDB
} 
post context of the desire expressions, since theories of narrative structure suggest that the evaluation points of a narrative can precede the expression of the events, goals and desires of the narrator (Labov, 1972; Swanson et al., 2014).

Our approach builds on seminal work on a computational model of Lehnert's plot units, that applied modern NLP tools to tracking narrative affect states in Aesop's Fables (Goyal et al., 2010; Lehnert, 1981; Goyal and Riloff, 2013). Our framing of the problem is also inspired by recent work that identifies three forms of desire expressions in short narratives from MCTest and SimpleWiki and develops models to predict whether desires are fulfilled or unfulfilled (Chaturvedi et al., 2016). However DesireDB's narrative and sentence structure is more complex than either MCTest or SimpleWiki (Richardson et al., 2013; Coster and Kauchak, 2011).

We propose new features ( $\operatorname{Sec} 4.1$ ), as well as testing features used in previous work, and apply different classifiers to model desire fulfillment in our corpus. We also directly compare to results on MCTest and SimpleWiki (Sec 4.4). We apply LSTM models that distinguish between prior and post context and capture the flow of the narrative. Our best system, a Skip-Thought RNN model, achieves an F-measure of 0.70 , while a logistic regression system achieves 0.66. Our models and features outperform Chaturvedi et al. (2016) on MCTest and SimpleWiki, while providing new results for a new corpus for tracking desires in first-person narratives. Moreover, analysis of our results shows that features representing the discourse structure (such as overt discourse relation markers) are the best predictors of fulfillment status of a desire or goal. We also show that both prior and post context are important for this task.

We discuss related work in Sec. 2 and describe our corpus and annotations in Sec. 3. Section 4 presents our features and methods for modeling desire fulfillment in narratives along with the experiments and results including comparison to previous work. Finally, we present conclusions and future directions in Sec. 5.

\section{Related Work}

There has recently been an upsurge in interest in computational models of narrative structure (Lehnert, 1981; Wilensky, 1982) and story understanding (Rahimtoroghi et al., 2016; Swanson
Prior-Context: (1) I ran the Nike+ human Race 10K new York in under 57 minutes! (2) Then at the all-American rejects concert, I somehow ended up right next to this really cute guy and he seemed interested in me. (3) Was I imagining things? He was really nice; (4) I dropped something and it was dark, he bent with his cell phone light to help me look for it. (5) We spoke a little, but it was loud and not suited for conversation there.

Desire-Expression-Sentence: I [had hoped to] ask him to join me for a drink or something after the show (if my courage would allow such a thing) but he left before the end and I didn't see him after that.

Post-Context: (1) Maybe I'll try missed connections lol. (2) I didn't want to tell him I think he's cute or make any gay references during the show because if I was wrong that would make standing there the whole rest of the concert too awkward... (3) Afterward, I wandered through the city making stops at several bars and clubs, met some new people, some old people (4) As in people I knew - I actually didn't met any old people, unless you count the tourist family whose dad asked me about my t-shirt. (5) And when I thought the night was over (and the doorman of the club did insist it was over) I met this great guy going into the subway.

Figure 2: A desire expression with its surrounding context extracted from a personal narrative

et al., 2014; Ouyang and McKeown, 2015, 2014). However there has been limited work on computational models for recognizing the expression of the protagonist's goals and desires in narrative genres.

Our approach builds on work by Goyal and Riloff (2013) that applied modern NLP tools to track narrative affect states in Aesop's Fables (Goyal et al., 2010). They present a system called AESOP that uses a number of existing resources to identify affect states of the characters as part of deriving plot units. The motivation of modeling plot units is the idea that emotional reactions are central to the notion of a narrative and the main plot of a story can be modeled by tracking the transition between the affect states (Lehnert, 1981). The AESOP system identifies affect states and creates links between them to model plot units and is evaluated on a small set of two-character fables. They performed a manual annotation to examine different types of affect expressions in the narratives. Their study shows that many affect states arise from events where a character is acted upon in positive or negative ways, not explicit expression of emotions. They also show that most of the affect states emerge by the expression of goals and plans and goal completion. Some of our features are motivated by the idea that implicit sentiment polarity can represent success or failure of goals and can be used to better model desire and goal 
fulfillment in a narrative (Reed et al., 2017), although we cannot directly compare our findings to theirs because their annotations are not publicly available.

Chaturvedi et al. (2016) exploit two deliberately simplified datasets in order to model desire and its fulfillment: MCTest which contains 660 stories limited to content understandable by 7-year old children, and, SimpleWiki created from a dump of the Simple English Wikipedia discarding all the lists, tables and titles. They use desire statements matching a list of three verb phrases, wanted to, hoped to, and wished to. Their context representation consists of five or fewer sentences following the desire expression. They use BOW (Bag of Words) as baseline and apply unstructured and structured models for desire fulfillment modeling with different features motivated by narrative structure. Their best result is achieved with a structured prediction model called Latent Structured Narrative Model (LSNM) which models the evolution of the narrative by associating a latent variable with each fragment of the context in the data. Their best unstructured model is a Logistic Regression classifier that uses all of their features.

Recent work on computational models of semantics provides an evaluation test for story understanding (Mostafazadeh et al., 2017). The task includes four-sentence stories, each with two possible endings where only one is correct. The goal is for each system to select the correct ending of the story by modeling different levels of semantics in narratives, such as lexical, sentential and discourse-level. The highest performing model with $75 \%$ accuracy used a linear regression classifier with several features such as neural language models and stylistic features to model the story coherence (Schwartz et al., 2017). The results from other systems showed that sentiment is an important factor and using only sentiment features could achieve about $65 \%$ accuracy on the test.

\section{DesireDB Corpus}

DesireDB aims to provide a testbed for modeling desire and goals in personal narrative and predicting their fulfillment status. We develop a systematic method to identify desire and goal statements, and then collect annotations to create goldstandard labels of fulfillment status as well as spans of text marked as evidence.

\subsection{Identifying Desires and Goals}

Our corpus is a subset of the Spinn3r corpus (Burton et al., 2011, 2009), consisting of firstperson narratives from six personal blog domains: livejournal.com, wordpress.com, blogspot.com, spaces.live.com, typepad.com, travelpod.com. To create our dataset, we select only desire expressions involving some version of the first-person. In first-person narratives, the narrator and protagonist naturally align which makes it much easier to identify and track the protagonist than in fiction or historical genre. Thus, selecting narrative passages with expressions of desire relating to the first-person are very likely to discuss subsequent behaviors to achieve that desire and the end result. Put simply, zooming in on first-person desires means that desire and its aftermath are more likely to be highly topical for the narrative. This corpus, then, is highly suitable as a testbed for modeling human desires and their fulfillment.

Human desires and goals can be expressed linguistically in many different ways, including both explicit verbal and nominal markers of desire or necessity (e.g., want, hope) and more general markers of urges (e.g., craving, hunger, thirst). To systematically discover predicates that specify desires, we browsed FrameNet 1.7 (Baker et al., 1998) selecting frames that seemed likely to contain lexical units specifying desires: Beingnecessary, Desiring, Have-as-a-demand, Needing, Offer, Purpose, Request, Required-event, Scheduling, Seeking, Seeking-to-achieve, Stimulus-focus, Stimulate-emotion, and Worry. We then selected 100 representative instances of that frame in English Gigaword (Parker et al., 2011) by first selecting the 10 most frequent lexical units in that frame, and then selecting 10 random instances per lexical unit. One of the authors examined each set of 100 instances, estimating for each sentence whether the predicate specifies a goal that the surrounding text picks up on. Because we were looking for predicates that reliably specify desires that motivate a protagonist's actions, we eliminated frames where less than $80 \%$ of the sentences showed this characteristic.

This resulted in a downsample to the following four frames: Desiring, Needing, Purpose, and Request. We selected only the verbal lexical units because we found that verbs were more likely to introduce goals than nouns or adjectives. We examined 100 instances for each verbal lex- 


\section{Data-Instance:}

Prior-Context: ConnectiCon!!! Ya baby, we did go this year as planned! Though this year we weren't in the artist colony, so I didn't see much point in posting about it before hand.

Desire-Expression-Sentence: This year we [wanted to] be part of the main crowd.

Post-Context: We wanted to get in on all the events and panels that you cant attend when watching over a table. And this year we wanted to cosplay! My hubby and I decided to dress up like aperture Science test subjects from the PC game portal. It was a good and original choice, as we both ended up being the only portal related people in the con (unless there were others who came late in the evening we didn't see) It was loads of fun and we got a surprising amount of attention.

\begin{tabular}{l}
\hline Annotations: \\
Fulfillment-Label: Fulfilled \\
Fulfillment-Agreement-Score: 3 \\
Evidence: Though this year we weren't in the artist \\
colony. We wanted to get in on all the events and pan- \\
els that you cant attend when watching over a table. \\
Evidence-Overlap-Score: 3
\end{tabular}

Figure 3: Example of data in DesireDB

ical unit, discarding as before. This resulted in 37 verbs. For each verb, we systematically constructed and coded all past forms of the verb (e.g., was [verb]ing, had [verb]ed, had been [verb]ing, [verb]ed, didn't [verb], etc.) because we posited that morphological form itself may convey likelihood of fulfillment (e.g., a past perfect I had wanted to ... signals that something changed, either the desire or fulfillment). We initially experimented with both past and (historical) present, but past tense verb patterns resulted in much higher precision. We counted the instances of these patterns in our dataset, and retained only those lemmas with at least 1000 instances across the corpus.

We extract stories containing the verbal patterns of desire, with five sentences before and after the desire expression sentence as context (See Fig. 2). Our annotation results provide support that the evidence of desire fulfillment can be expressed before the desire statement. We also study the effect of prior and post context in understanding desire fulfillment in our experiments (Section 4) and show that using the narrative context preceding the desire statement improves the results.

\subsection{Data Annotation}

We extracted $\sim 600 \mathrm{~K}$ desire expressions with their context, and then sample 3,680 instances for annotation. This subset consists of 16 verbal patterns (when collapsing all morphological forms to their

\begin{tabular}{lccccc}
\hline Pattern & Count & Ful & Unf & Unk & None \\
\hline wanted to & 2,510 & $49 \%$ & $35 \%$ & $14 \%$ & $2 \%$ \\
needed to & 202 & $65 \%$ & $16 \%$ & $16 \%$ & $3 \%$ \\
ordered & 201 & $71 \%$ & $21 \%$ & $6 \%$ & $2 \%$ \\
arranged to & 199 & $68 \%$ & $13 \%$ & $16 \%$ & $3 \%$ \\
decided to & 68 & $87 \%$ & $9 \%$ & $4 \%$ & $0 \%$ \\
hoped to & 68 & $19 \%$ & $68 \%$ & $12 \%$ & $1 \%$ \\
couldn't wait & 68 & $79 \%$ & $3 \%$ & $15 \%$ & $3 \%$ \\
wished to & 66 & $27 \%$ & $35 \%$ & $30 \%$ & $8 \%$ \\
scheduled & 60 & $43 \%$ & $25 \%$ & $27 \%$ & $5 \%$ \\
asked for & 60 & $53 \%$ & $27 \%$ & $15 \%$ & $5 \%$ \\
required & 58 & $69 \%$ & $16 \%$ & $15 \%$ & $0 \%$ \\
requested & 30 & $60 \%$ & $20 \%$ & $20 \%$ & $0 \%$ \\
demanded & 30 & $60 \%$ & $23 \%$ & $17 \%$ & $0 \%$ \\
ached to & 20 & $50 \%$ & $40 \%$ & $10 \%$ & $0 \%$ \\
aimed to & 20 & $55 \%$ & $30 \%$ & $15 \%$ & $0 \%$ \\
desired to & 20 & $50 \%$ & $25 \%$ & $25 \%$ & $0 \%$ \\
\hline Total & 3,680 & $53 \%$ & $31 \%$ & $14 \%$ & $2 \%$ \\
\hline
\end{tabular}

Table 1: Distribution of desire verbal patterns and fulfillment labels in DesireDB

head word). A group of pre-qualified Mechanical Turkers then labelled each instance. The annotators labelled the fulfillment status of the desire expression sentence based on the prior and post context, by choosing from three labels: Fulfilled, Unfulfilled, and Unknown from the context. They were also asked to mark the evidence for the label they had chosen by specifying a span of text in the narrative. For each data instance, we asked the Turkers to mark the subject of the desire expression and determine if the expressed desire is hypothetical (e.g., a conditional sentence) or not.

The annotators were selected from a list of prequalified workers who had successfully passed a test on a textual entailment task with $100 \%$ correct answers. They were provided with detailed instructions and examples as to how to label the desires and mark the evidence. We also specified the desire expression verbal pattern using square brackets (as shown in Fig. 1 and 2) for more clarity. Three annotators were assigned to work on each data instance. To generate the gold-standard labels we used majority vote and the cases with no agreement were labeled as 'None'.

Table 1 reports the distribution of data and goldstandard labels (Ful:Fulfilled, Unf:Unfulfilled, Unk:Unknown from the context). About half of the desire expressions (53\%) were labeled Fulfilled and about one third (31\%) were labeled $U n$ fulfilled. The annotators didn't agree on about $2 \%$ of the instances, that were labeled None. As Tabel 1 shows, the distribution of labels is not uniform across different verbal patterns. For in- 
stance, decided to and couldn't wait are highly skewed towards Fulfilled as opposed to hoped to which includes $68 \%$ Unfulfilled instances. Some patterns seem to be harder to annotate, like wished to, which has the highest rate of Unknown (30\%) and None (8\%) among all.

Other than fulfillment status, for each data instance in our corpus we include the agreementscore which is the number of annotators that agreed on the assigned label. In addition, we provide the evidence as a part of the DesireDB data, by merging the text spans marked by the annotators as evidence. We compared the evidence spans pairwise to measure the overlap-score, indicating the number of pairs of annotators with overlapping responses. An example is shown in Figure 3. The first part is the extracted data including the desire expression with prior and post context, and the second part is the gold-standard annotations.

To assess inter-annotator agreement for Fulfillment, we calculated Krippendorff-alpha Kappa (Krippendorff, 1970, 2004) for pairwise inter-annotator reliability, and, the average of Kappa between each annotator and the majority vote. These two metrics are 0.63 and 0.88 respectively. Overall, $66 \%$ of the data was labeled with total agreement (where all three annotators agreed on the same label) and about $32 \%$ of data was labeled by two agreements and one disagreement. We also examined the agreements across each label separately. For Fulfilled class, total agreement rate is $75 \%$, which for Unfulfilled is $67 \%$, and on Unknown from the context is $41 \%$. We believe this indicates that annotating unfulfilled desires was harder than fulfilled cases. For evidence marking, in $79 \%$ of the data all three annotators marked overlapping spans.

\section{Modeling Desire Fulfillment}

We conducted a range of experiments on predicting fulfillment status of desires and goals, using different features and models, including LSTM architectures that can encode the sequential structure of the narratives. We first describe our features and models. Then, we present our feature analysis study to examine their importance in modeling fulfillment. Finally we provide results of direct comparison to previous work on the existing corpora.
Sentiment: Negative

Prior-Context(4): "I had been working for hours on boring paperwork and financial stuff, and I was really crabby."

Sentiment: Negative

Prior-Context(5): I decided it was time to take a break and thought, should I read a magazine or watch best Week Ever?

Sentiment: Negative

Desire-Epxression-Sentence: But I realized that what I really [wanted to] do was go for a run!

Sentiment: Positive

Post-Context(1): That was pretty amazing, to transition mentally from 'having to' to 'wanting to' run.

Sentiment: Positive

Post-Context(2): So I did a quick, fun 2.75 miles.

Figure 4: Example of sentiment features, where prior context is negative while the post context is positive, implying fulfillment of the desire

\subsection{Features Description}

In our original informal examination of the DesireDB development data, we noticed several ways that a writer can signal (lack of) fulfillment of a desire like "I hoped to pick up a dictionary". First, they may mention an outcome that entails ("The book I bought was...") or strongly implies fulfillment ("I went back home happily."). However, we noticed that in many cases of fulfillment, the 'marker' was simply the absence of any mention that things went wrong. For lack of fulfillment, while we found cases where writers explicitly state that their desire wasn't met, we noted many instances where evidence came from mentioning that an enabling condition for fulfillment wasn't met ("The bookstore was closed.").

True machine understanding of these kinds of narrative structures requires robust models of the complex interplay of semantics (including negation) as well as world knowledge about the scripts for tasks like buying books, including what count as enabling conditions and entailers for fulfillment. While we hope to explore more articulated models in the future, for our experiments we considered reasonable proxies for the conditions mentioned above using existing resources (note that we also tested LSTM models described below, which may implicitly learn such relationships with sufficient data). One set (Desire Features) indexes properties of the desire expression (e.g., the desire verb) as well as overlap between the desired object/event and the surrounding context. The remaining features attempt to find general markers 
for success or failure. One set (Discourse Features) looks for overt discourse relation markers that signal violation of expectation (e.g., 'but', 'however') or its opposite (e.g., 'so'). Another uses the Connotation Lexicon (Feng et al., 2013) to model whether the context provides a positive or negative event. All of these features are inspired by Chaturvedi et al. (2016). Finally, motivated by the AESOP modeling of affect states for identifying plot units (Goyal and Riloff, 2013), one set of features (Sentiment-Flow-Features) indexes whether there has been a change in sentiment in the surrounding context (which might be the mention of a thwarted effort or a hard won victory). Figure 4 provides an example of this.

In addition to a BOW (Bag of Words) baseline, we extracted the four types of features mentioned above. For features that examine the context around the desire expression, our experiments used the pre-context, the post-context, or both, as discussed below; context features are computed per sentence $i$ of the context. We also tested various ablations of these features described below as well. We now describe the full set of features in more detail.

Desire-Features. From a desire expression of the form ' $X$ Ved S', we extract the lexical feature Desire-Verb, the lemma for V. We also extract a list of focal words, the content words in embedded sentence S. In Figure 4, these are 'do', 'go', and 'run'. The features Focal$\{$ Word,Synonym,Antonym $\}-M e n t i o n-i$ counts how many times each word, its synonyms, or its antonyms in WordNet (Fellbaum, 1998) are in the context, respectively. Similarly, Desire-SubjectMention- $i$ marks if subject $\mathrm{X}$ is mentioned in the context. Finally, boolean First-Person-Subject indicates if $\mathrm{X}$ is first person ('I', 'we').

Discourse-Features. This class of features count how many of two classes of discourse relation markers (Violated-Expectation- $i$ vs. MeetingExpectation-i) occur in the context. For the classes, we manually coded all overt discourse relation markers in the Penn Discourse Treebank three ways(violation, meeting, or neutral), leading to 15 meeting markers ('accordingly', 'so', 'ultimately', 'finally') and 31 violating ('although', 'rather', 'yet', 'but'). In addition, we also tracked the presence of the most frequent of these ('so' and 'but', respectively) in the desire sentence itself by the booleans So-Present and But-Present.

\begin{tabular}{ccccc}
\hline Fulfilled & Unfulfilled & Unknown & None & Total \\
\hline 1,366 & 953 & 380 & 70 & 2,780 \\
\hline
\end{tabular}

Table 2: Simple-DesireDB dataset

Connotation-Features. Beyond the use of WordNet expansion for Focal-Word-Mention-i, we also used the Connotation Lexicon (Feng et al., 2013), a lexical resource marking very general connotation polarities (positive or negative) of words (as opposed to more specific sentiment lexicons). Connotation-Agree-i counts for each word $w$ in focal words the number of words in the context that have the same connotation polarity as $w$. Connotation-Disgree- $i$ is defined similarly.

Sentiment-Flow-Features. To model affect states, we compute a sentiment score for the desire expression sentence as well as each sentence in the context. Then for each sentence of the context, the booleans Sentiment-Agree-i and SentimentDisagree-i mark whether that sentence and the desire expression sentence have the same sentiment polarity (see Figure 4). While there is evidence suggesting that models of implicit sentiment (e.g., (Goyal et al., 2010; Reed et al., 2017)) could do much better at tracking affect states, here we use the Stanford Sentiment system (Socher et al., 2013).

\subsection{LSTM Models}

Our features are motivated by narrative characteristics but do not directly capture the sequential structure of the narratives. We thus apply neural network models suitable for sequence learning, in order to directly encode the order of the sentences in the story and distinguish between prior and post context. We use two different architectures of LSTM (Long Short-Term Memory) (Hochreiter and Schmidhuber, 1997) models to generate sentence embeddings and then apply a three-layer RNN (Recurrent Neural Network) for classification. We used Keras (Chollet, 2015) as a deep learning toolkit for implementing our experiments.

Skip-Thoughts. This is a sequential model that uses pre-trained skip-thoughts model (Kiros et al., 2015) as the embedding of sentences. It first concatenates features, if any, with embeddings, and then uses LSTM to generate a single representation for the context sequence, which is the output of the last unit. That single representation is then 


\begin{tabular}{l|l|ccc|ccc|ccc}
\hline Method & Features & Ful-P & Ful-R & Ful-F1 & Unf-P & Unf-R & Unf-F1 & Precision & Recall & F1 \\
\hline \multirow{2}{*}{ Skip-Thought } & BOW & 0.75 & 0.70 & 0.72 & 0.54 & 0.61 & 0.57 & 0.65 & 0.65 & 0.65 \\
& ALL & $\mathbf{0 . 8 0}$ & $\mathbf{0 . 7 1}$ & $\mathbf{0 . 7 5}$ & $\mathbf{0 . 5 9}$ & $\mathbf{0 . 7 0}$ & $\mathbf{0 . 6 4}$ & $\mathbf{0 . 7 0}$ & $\mathbf{0 . 7 0}$ & $\mathbf{0 . 7 0}$ \\
\hline CNN-RNN & BOW & 0.75 & 0.73 & 0.74 & 0.57 & 0.60 & 0.58 & 0.66 & 0.66 & 0.66 \\
& ALL & 0.75 & 0.79 & 0.77 & 0.61 & 0.56 & 0.59 & 0.68 & 0.68 & 0.68 \\
\hline
\end{tabular}

Table 3: Results of LSTM models on Simple-DesireDB

\begin{tabular}{l|ccc|ccc|ccc}
\hline Data & Ful-P & Ful-R & Ful-F1 & Unf-P & Unf-R & Unf-F1 & Precision & Recall & F1 \\
\hline Desire & 0.74 & 0.75 & 0.75 & 0.57 & 0.56 & 0.57 & 0.66 & 0.66 & 0.66 \\
Desire+Prior & 0.78 & 0.73 & 0.75 & 0.58 & 0.65 & 0.61 & 0.68 & 0.69 & 0.68 \\
Desire+Post & 0.76 & 0.70 & 0.73 & 0.55 & 0.62 & 0.59 & 0.66 & 0.66 & 0.66 \\
Desire+Context & $\mathbf{0 . 8 0}$ & $\mathbf{0 . 7 1}$ & $\mathbf{0 . 7 5}$ & $\mathbf{0 . 5 9}$ & $\mathbf{0 . 7 0}$ & $\mathbf{0 . 6 4}$ & $\mathbf{0 . 7 0}$ & $\mathbf{0 . 7 0}$ & $\mathbf{0 . 7 0}$ \\
\hline
\end{tabular}

Table 4: Results of Skip-Thought using different parts of data, with ALL features on Simple-DesireDB

concatenated with embedding-feature concatenation of desire sentence and is fed into a multi-layer network to yield a single binary output.

CNN-RNN. The only difference between the CNN-RNN model and Skip-Thought is that it uses the 1-dimensional convolution with maxover-time pooling introduced in (Kim, 2014) to generate the sentence embedding from word embedding, instead of using skip-thoughts. We use Google News Vectors (Mikolov et al., 2013) for the word embedding with different sizes from 1 to 7 for the kernel.

For our experiments, we first constructed a subset of DesireDB that we will call SimpleDesireDB, in order to be able to compare more directly to the models and data used in previous work. Chaturvedi et al. (2016) used three verb phrases to identify desire expressions (wanted to, hoped to, and wished to), so we selected a portion of our corpus including these patterns along with two other expressions (couldn't wait to and decided to) to have sufficient data for experiments. Table 2 shows the distribution of labels in this subset. For classification experiments we use data labeled as Fulfilled and Unfulfilled, thus the majority class accuracy is $59 \%$. We split the data into Train (1,656), Dev (327), and Test (336) sets for the experiments.

Results of our two LSTM models for Fulfilled (Ful) and Unfulfilled (Unf) classes and the overall classification task (P:precision, R:recall) on Simple-DesireDB are presented in Table 3. ALL feature set includes all the features described in Sec. 4.1 (without BOW). The results indicate that our features can considerably improve the model, compared to the BOW baseline (F1 improved from
0.65 to 0.70 for Skip-Thought). We also conducted 4 sets of experiments to study the importance of prior, post and the whole context in predicting fulfillment status, using our best model. The results of Skip-Thought using different contextual representations are in Table 4 with ALL features. The results indicate that adding features from prior context alone improves the results. The best results are obtained by including the whole context and desire sentence.

We then experimented with our best model on all of DesireDB. We also trained Naive Bayes, SVM and Logistic Regression (LR) classifiers as baselines, with the best results on the Dev set achieved by Logistic Regression. Table 5 shows the results of Skip-Thought and LR on DesireDB for different features on the test set. Our feature ablation study on the Dev set, discussed in Sec. 4.3, indicates that Discourse features are better predictors of fulfillment status, so we present results using only Discourse features in addition to BOW and ALL.

All of the results indicate that similar features and methods achieve better results for the Fulfilled class as compared to Unfulfilled. We believe the reason is that identifying unfulfillment of a desire or goal is a more difficult task, as discussed in the annotation description in Section 3.2. To further our analysis on the annotation disagreements, we examined the cases where only two annotators agreed on the assigned label. From the expressions labeled Fulfilled by two annotators, $64 \%$ were labeled Unknown from the context by the disagreeing annotator, and only $36 \%$ were labeled Unfulfilled. However, these numbers for the Unfulfilled class are respectively $49 \%$ and $51 \%$, indicating a 


\begin{tabular}{l|l|ccc|ccc|ccc}
\hline Method & Features & Ful-P & Ful-R & Ful-F1 & Unf-P & Unf-R & Unf-F1 & Precision & Recall & F1 \\
\hline Skip- & BOW & 0.78 & 0.78 & 0.78 & 0.57 & 0.56 & 0.57 & 0.67 & 0.67 & 0.67 \\
Thought & All & 0.78 & 0.79 & 0.79 & 0.58 & 0.56 & 0.57 & 0.68 & 0.68 & 0.68 \\
& Discourse & $\mathbf{0 . 8 0}$ & $\mathbf{0 . 7 9}$ & $\mathbf{0 . 8 0}$ & $\mathbf{0 . 6 0}$ & $\mathbf{0 . 6 0}$ & $\mathbf{0 . 6 0}$ & $\mathbf{0 . 7 0}$ & $\mathbf{0 . 7 0}$ & $\mathbf{0 . 7 0}$ \\
\hline Logistic & BOW & 0.69 & 0.65 & 0.67 & 0.53 & 0.57 & 0.55 & 0.61 & 0.61 & 0.61 \\
Regression & All & 0.79 & 0.70 & 0.74 & 0.52 & 0.64 & 0.58 & 0.66 & 0.67 & 0.66 \\
& Discourse & 0.75 & 0.84 & 0.80 & 0.60 & 0.45 & 0.52 & 0.67 & 0.65 & 0.66 \\
\hline
\end{tabular}

Table 5: Results of best LSTM model with different feature sets, compared to LR on DesireDB

\begin{tabular}{l|ccc}
\hline Features & Precision & Recall & F1 \\
\hline ALL & 0.64 & 0.64 & 0.64 \\
Discourse & 0.66 & 0.64 & 0.65 \\
But-Present & $\mathbf{0 . 7 2}$ & $\mathbf{0 . 6 4}$ & $\mathbf{0 . 6 8}$ \\
ALL w/o But-Present & 0.58 & 0.58 & 0.58 \\
\hline
\end{tabular}

Table 6: Results of Logistic Regression classifier with different feature sets on Simple-DesireDB

stronger disagreement between annotators when labeling Unfulfilled expressions.

\subsection{Feature Selection Experiments}

We used the InfoGain measure to rank features based on their importance in modeling desire fulfillment. The top 5 features are: But-Present, Post-Context-Connotation-Disagree, Post-Context-Violated-Expectation, Desire-Verb, Is-First-Person. We also tested different feature sets separately. We describe our experiment results below.

The results of the feature ablation experiments using LR model are shown in Table 6. The ALL feature set includes all the features described in Sec. 4.1 (without BOW). We obtained high precision and F-measure using the Discourse features. We also experimented with our top feature from the InfoGain analysis, But-Present, which surprisingly achieves a high F-measure, compared to using ALL and Discourse feature sets. The last row of Table 6 shows the results of using ALL features excluding But-Present. This indicates that features motivated by narrative structure are primarily driving improvement. In previous work Chaturvedi et al. (2016) show that a model representing narrative structure could beat the BOW baseline, but they performed no systematic feature ablation. Our results suggest that ultimately, the presence of "but" is likely a central driver for their improvements as well.

\begin{tabular}{l|l|ccc}
\hline Dataset & Method & Precision & Recall & F1 \\
\hline \multirow{2}{*}{ MCTest } & BOW & 0.41 & 0.50 & 0.45 \\
& Unstruct-LR & 0.71 & 0.63 & 0.67 \\
& LSNM & 0.70 & 0.84 & 0.74 \\
& Discourse-LR & $\mathbf{0 . 6 3}$ & $\mathbf{0 . 8 3}$ & $\mathbf{0 . 7 1}$ \\
& SkipTh-BOW & $\mathbf{0 . 7 2}$ & $\mathbf{0 . 6 8}$ & $\mathbf{0 . 7 0}$ \\
& SkipTh-ALL & $\mathbf{0 . 7 0}$ & $\mathbf{0 . 8 4}$ & $\mathbf{0 . 7 6}$ \\
\hline Simple & BOW & 0.28 & 0.20 & 0.23 \\
Wiki & Unstruct-LR & 0.50 & 0.09 & 0.15 \\
& LSNM & 0.38 & 0.21 & 0.27 \\
& Discourse-LR & $\mathbf{0 . 3 2}$ & $\mathbf{0 . 8 2}$ & $\mathbf{0 . 4 6}$ \\
& SkipTh-BOW & $\mathbf{0 . 7 1}$ & $\mathbf{0 . 2 6}$ & $\mathbf{0 . 3 8}$ \\
& SkipTh-ALL & $\mathbf{0 . 3 3}$ & $\mathbf{0 . 1 6}$ & $\mathbf{0 . 2 2}$ \\
\hline
\end{tabular}

Table 7: Previous work and our results for the Fulfilled class, on MCTest and SimpleWiki.

\subsection{Comparison to Previous Work}

We directly compare our methods and features to the most relevant previous work (Chaturvedi et al., 2016). They applied their models on two datasets and reported the results for the Fulfilled class. We present the same metrics in Table 7, using our best model Skip-Thought (SkipTh). We also present results of our LR model with our Discourse features, Discourse-LR, trained and tested on their corpora to compare to their features. The first three rows show the results from Chaturvedi et al. (2016) for comparison. As described in Sec. 2, they used BOW as baseline, LSNM is their best model, and Unstruct-LR is their unstructured model that uses all of their features with LR.

On both corpora, Discourse-LR outperforms Unstruct-LR, showing that the Discourse features are stronger indicators of the desire fulfillment status when used with LR classifier. In addition, on SimpleWiki, LR-Discourse outperforms their structured model, LSNM (0.46 vs. 0.27 on F-1).

\section{Conclusion and Future Work}

We created a novel dataset, DesireDB, for studying the expression of desires and their fulfillment in narrative discourse. We show that contextual 
features help with classification, and that both prior and post context are useful. Finally, we show that exploiting narrative structure is helpful, both directly in terms of the utility of discourse relation features and indirectly via the superior performance of a Skip-Thought LSTM model.

In future work, we plan to explore richer features and models for semantic and discourse-based features, as well as the utility of more narrativelyaware features. For instance, the sentiment flow features roughly track the notion that the arc of a narrative may implicitly reveal resolution of a goal via changes in affect states. We hope to examine whether there are other similar rough-grained measures of change over the entire narrative that can improve the results.

DesireDB contains annotator-labeled spans for evidence for the annotator's conclusions. While we have not used this labeling, we plan to use it in future work. Finally, we hope to turn to automatically detecting instances of desire expressions that give rise to the kind of goal-oriented narratives DesireDB contains. Here we have used highprecision search patterns but our annotations show that such patterns still admitted 134 hypothetical desires (e.g., 'If I had wanted to buy a book'). It would appear that distinguishing hypothetical vs. real desires itself could be an interesting problem.

\section{Acknowledgments}

This research was supported by Nuance Foundation Grant SC-14-74, NSF Grant IIS-1302668-002 and IIS-1321102.

\section{References}

C.F. Baker, C.J. Fillmore, and J.B. Lowe. 1998. The berkeley framenet project. In Proceedings of the 36th Annual Meeting of the Association for Computational Linguistics and 17th International Conference on Computational Linguistics-Volume 1. Association for Computational Linguistics, pages 86-90.

Allan Bell. 2005. News stories as narratives. The Language of Time: A Reader page 397.

Jerome Bruner. 1991. The narrative construction of reality. Critical Inquiry 18:1-21.

Kevin Burton, Akshay Java, and Ian Soboroff. 2009. The ICWSM 2009 Spinn3r dataset. In Proceedings of the Third Annual Conference on Weblogs and Social Media (ICWSM 2009).

Kevin Burton, Niels Kasch, and Ian Soboroff. 2011. The icwsm 2011 spinn3r dataset. In Proceedings of the Annual Conference on Weblogs and Social Media (ICWSM).

Snigdha Chaturvedi, Dan Goldwasser, and Hal Daume III. 2016. Ask, and shall you receive? understanding desire fulfillment in natural language text. In Proceedings of the National Conference on Artificial Intelligence.

Francois Chollet. 2015. Keras.

William Coster and David Kauchak. 2011. Simple english wikipedia: a new text simplification task. In Proceedings of the 49th Annual Meeting of the Association for Computational Linguistics: Human Language Technologies: short papers-Volume 2. Association for Computational Linguistics, pages 665669.

David K. Elson. 2012. Modeling Narrative Discourse. Ph.D. thesis, Columbia University, New York City.

Christiane Fellbaum. 1998. WordNet: An Electronic Lexical Database. MIT Press.

Song Feng, Jun Seok Kang, Polina Kuznetsova, and Yejin Choi. 2013. Connotation lexicon: A dash of sentiment beneath the surface meaning. In Association for Computational Linguistics (ACL).

Andrew Gordon, Cosmin Bejan, and Kenji Sagae. 2011. Commonsense causal reasoning using millions of personal stories. In Twenty-Fifth Conference on Artificial Intelligence (AAAI-11).

Amit Goyal and Ellen Riloff. 2013. A computational model for plot units. Computational Intelligence 29(3):466-488.

Amit Goyal, Ellen Riloff, and Hal Daumé III. 2010. Automatically producing plot unit representations for narrative text. In Proceedings of the 2010 Conference on Empirical Methods in Natural Language Processing. pages 77-86.

Sepp Hochreiter and Jürgen Schmidhuber. 1997. Long short-term memory. Neural computation 9(8):1735-1780.

Dan Jurafsky, Victor Chahuneau, Bryan R Routledge, and Noah A Smith. 2014. Narrative framing of consumer sentiment in online restaurant reviews. First Monday 19(4).

Yoon Kim. 2014. Convolutional neural networks for sentence classification. arXiv preprint arXiv:1408.5882 .

Ryan Kiros, Yukun Zhu, Ruslan R Salakhutdinov, Richard Zemel, Raquel Urtasun, Antonio Torralba, and Sanja Fidler. 2015. Skip-thought vectors. In Advances in neural information processing systems. pages 3294-3302.

Klaus Krippendorff. 1970. Bivariate agreement coefficients for reliability of data. Sociological methodology 2:139-150. 
Klaus Krippendorff. 2004. Content analysis: An introduction to its methodology. Sage.

William Labov. 1972. The transformation of experience in narrative syntax. In Language in the Inner City, University of Pennsylvania Press, Philadelphia, pages 354-396.

Wendy G Lehnert. 1981. Plot units and narrative summarization. Cognitive Science 5(4):293-331.

Dan P McAdams, Ruthellen Ed Josselson, and Amia Ed Lieblich. 2006. Identity and story: Creating self in narrative.. American Psychological Association.

Tomas Mikolov, Ilya Sutskever, Kai Chen, Greg S Corrado, and Jeff Dean. 2013. Distributed representations of words and phrases and their compositionality. In Advances in neural information processing systems. pages 3111-3119.

Nasrin Mostafazadeh, Michael Roth, Annie Louis, Nathanael Chambers, and James F Allen. 2017. Lsdsem 2017 shared task: The story cloze test. $L S D$ Sem 2017 page 46 .

Katherine Nelson. 1989. Narratives from the Crib. University Press, Cambridge, MA.

Jessica Ouyang and Kathleen McKeown. 2015. Modeling reportable events as turning points in narrative. In EMNLP. pages 2149-2158.

Jessica Ouyang and Kathy McKeown. 2014. Towards automatic detection of narrative structure. In LREC. Citeseer, pages 4624-4631.

Robert Parker, David Graff, Junbo Kong, Ke Chen, and Kazuaki Maeda. 2011. English gigaword. Linguistic Data Consortium .

Livia Polanyi. 1989. Telling the American Story: A Structural and Cultural Analysis of Conversational Storytelling. MIT Press.

Elahe Rahimtoroghi, Ernesto Hernandez, and Marilyn A. Walker. 2016. Learning fine-grained knowledge about contingent relations between everyday events. In Proceedings of SIGDIAL 2016. pages 350-359.

D.N. Rapp and R.J. Gerrig. 2006. Predilections for narrative outcomes: The impact of story contexts and reader preferences. Journal of Memory and Language 54(1):54-67.

Lena Reed, Jiaqi Wu, Shereen Oraby, Pranav Anand, and Marilyn Walker. 2017. Learning lexicofunctional patterns for first-person affect. In Proceedings of the 55th annual meeting of the Association for Computational Linguistics (ACL-17). ACL.

Matthew Richardson, Christopher JC Burges, and Erin Renshaw. 2013. Mctest: A challenge dataset for the open-domain machine comprehension of text. In EMNLP. volume 3, page 4 .
Roy Schwartz, Maarten Sap, Ioannis Konstas, Leila Zilles, Yejin Choi, and Noah A Smith. 2017. Story cloze task: Uw nlp system. LSDSem 2017 page 52.

Richard Socher, Alex Perelygin, Jean Wu, Jason Chuang, Christopher D. Manning, Andrew Ng, and Christopher Potts. 2013. Recursive deep models for semantic compositionality over a sentiment treebank. In Proceedings of the 2013 Conference on Empirical Methods in Natural Language Processing. pages 1631-1642.

Reid Swanson, Elahe Rahimtoroghi, Thomas Corcoran, and Marilyn A Walker. 2014. Identifying narrative clause types in personal stories. In 15th Annual Meeting of the Special Interest Group on Discourse and Dialogue.

Avril Thorne and V. Nam. 2009. The storied construction of personality. In Kitayama S. and Cohen D., editors, Handbook of Cultural Psychology, pages 491-505.

Tom Trabasso and Paul van den Broek. 1985. Causal thinking and the representation of narrative events. Journal of Memory and Language 24:612-630.

Robert Wilensky. 1982. Points: A theory of the structure of stories in memory. In Wendy G. Lehnert and Martin H. Ringle, editors, Strategies for Natural Language Processing. 